\title{
Sensitivity to Interaural Time Differences with Combined Cochlear Implant and Acoustic Stimulation
}

\author{
Tom Francart, ${ }^{1} \mathrm{Jan}^{\mathrm{B}}$ Brokx, ${ }^{2}$ and Jan Wouters ${ }^{1}$ \\ ${ }^{1}$ ExpORL, Department Neurosciences, K.U.Leuven, $O$ E $N$ 2, Herestraat 49 bus 721, B-3000 Leuven, Belgium \\ ${ }^{2}$ Department of Otorhinolaryngology and Head $\mathcal{E}$ Neck Surgery, University Hospital Maastricht, Postbus 5800, 6202 AZ, \\ Maastricht, Netherlands
}

Received: 6 June 2008; Accepted: 7 October 2008; Online publication: 2 December 2008

\begin{abstract}
The interaural time difference (ITD) is an important cue to localize sound sources. Sensitivity to ITD was measured in eight users of a cochlear implant (CI) in the one ear and a hearing aid (HA) in the other severely impaired ear. The stimulus consisted of an electric pulse train of $100 \mathrm{pps}$ and an acoustic filtered click train. Justnoticeable differences (JNDs) in ITD were measured using a lateralization paradigm. Four subjects exhibited median JNDs in ITD of 156, 341, 254, and $91 \mu \mathrm{s}$; the other subjects could not lateralize the stimuli consistently. Only the subjects who could lateralize had average acoustic hearing thresholds at 1,000 and 2,000 $\mathrm{Hz}$ better than 100-dB SPL. The electric signal had to be delayed by $1.5 \mathrm{~ms}$ to achieve synchronous stimulation at the auditory nerves.
\end{abstract}

Keywords: cochlear implant, hearing aid, localization, bimodal stimulation, electric acoustic stimulation, interaural time difference

\section{INTRODUCTION}

When a sound source is located to one side of the head in the horizontal plane, the sound waves will reach the ear closer to the sound source first. The delay between both ears is called the interaural time difference (ITD).

Correspondence to: Tom Francart - ExpORL, Department Neurosciences $\cdot$ K.U.Leuven $\cdot \mathrm{O} \& \mathrm{~N}$ 2, Herestraat 49 bus 721, B-3000 Leuven, Belgium. Telephone: +32-16-330476; fax: +32-16-330486; email: tom.francart@med.kuleuven.be
The human auditory system can use the ITD as well as the interaural level difference (ILD) to localize sound sources. ITDs range from $0 \mu \mathrm{s}$ for a sound straight ahead to $690 \mu$ s for a sound at $90^{\circ}$ (Moore 2003). For highfrequency sounds, the ITD becomes ambiguous because the period is smaller than the available ITD. In that case, however, the auditory system can make use of ITDs in the envelope of the high-frequency signals (Henning 1974).

Changes in ITD of about $10 \mu$ s can be detected by normal-hearing $(\mathrm{NH})$ subjects in low-frequency sinusoids (Yost 1974). Above 1,500 Hz, this process breaks down (Yost et al. 1971). While performance with amplitude-modulated high-frequency sinusoids is worse than with pure tones, performance with socalled transposed stimuli is nearly at the same level as with pure tones (Bernstein and Trahiotis 2002). Transposed stimuli are generated by modulating a high-frequency carrier with a half wave rectified envelope, resulting in the same output of the auditory filters as for the corresponding low-frequency signal.

While it is suggested that listeners are not able to use envelope ITDs in low-frequency sounds, Bernstein and Trahiotis (1985) show that those ITDs do affect the lateral position of low-frequency targets. They suggest that the envelopes are seemingly undetectable because the fine-structure ITD is dominant at low frequencies.

When the carriers of modulated signals are interaurally discrepant, just-noticeable differences (JNDs) in ITD can still be measured, but performance breaks down rapidly with increasing interaural frequency difference (Nuetzel and Hafter 1981). Blanks et al. (2007) however show that, for a simpler psychophysical task or an animal model, there is sensitivity to ITD when the interaural frequency difference increases up to several octaves. 
For many signals, both ITD and ILD cues are available and at first sight they appear to be interchangeable. However, Hafter and Carrier (1972) show that they do yield different percepts and are thus not entirely interchangeable. Nevertheless, the perception of ITDs is influenced by ILD; the JND in ITD is lowest when the ILD is zero (Domnitz 1973; Shepard and Colburn 1976).

Recent studies have shown that users of bilateral cochlear implants (CIs) are sensitive to ITDs, although much less than NH listeners. Best JNDs reported for pulse trains of about $100 \mathrm{pps}$ are around $100-350 \mu$ s and for higher pulse rates JNDs are much higher or immeasurable. JNDs in ITD were measured either through clinical speech processors (Laback et al. 2004; Senn et al. 2005) or with direct computercontrolled stimulation (Long et al. 2003; Majdak et al. 2006; van Hoesel 2004, 2007; Laback et al. 2007; van Hoesel and Tyler 2003; Lawson et al. 1998).

For CI users with residual hearing in the contralateral ear (bilateral bimodal stimulation), combined electric acoustic stimulation may be a feasible alternative to bilateral cochlear implantation. With clinical devices, it has been demonstrated that a contralateral hearing aid improves sound source localization and speech perception performance, as illustrated in a recent review by Ching et al. (2007). While performance improves by adding a contralateral hearing aid (HA), it is still poor compared to NH listeners. In this study, we focus on the fundamental understanding of localization using a CI combined with an HA.

In our previous study (Francart et al. 2008a), we demonstrated that users of bilateral bimodal hearing systems are sensitive to ILDs. In the current study, we focus on ITD perception by users of bimodal hearing systems. By means of a lateralization task using ITD cues, the JND in ITD is determined and the delay necessary to synchronize the CI and HA psychoacoustically is derived.

While localization performance improves when adding an HA to a contralateral CI, users of a clinical bimodal hearing system can most probably not perceive ITD cues. This is due to (1) the signal processing in the CI speech processor, (2) the tonotopic mismatch in stimulation between both ears, and (3) differences in processing delay between both ears. In this study, these technical issues were bypassed and the stimuli were optimized as to achieve maximal lateralization performance using ITD cues.

\section{METHODS}

\section{Apparatus}

Our test setup consisted of version 3 of the APEX program developed at ExpORL (K.U.Leuven;
Francart et al. 2008b), connected to an RME Hammerfall DSP sound card and an L34 experimental processor provided by Cochlear Ltd. An interface to the L34 was implemented in APEX and subsequently used to communicate with the L34. The L34 is part of the Cochlear NIC system. It is a modified speech processor that allows custom pulse sequences to be sent from a personal computer to the implant.

The first channel of the sound card was used to drive an ERA 3A insert phone and the second channel was used to trigger the start of the electric stimulation. In this way, the electric and acoustic signals were synchronized with 200-ns accuracy. Also, the clocks of the sound card and the L34 were synchronized to avoid drifting during stimulation. Both aspects were verified using an implant in a box and an oscilloscope.

The insert phone was calibrated using a $2-\mathrm{cm}^{3}$ coupler conforming to the ISO389 norm. The maximum distortion component that we measured for pure tones at all intensities used in this study was $43 \mathrm{~dB}$ below the sound pressure level (SPL) of the main spectral component. The maximum sound level that was presented to the subjects was 112-dB SPL. The shapes of both the electric and acoustic signals were checked using an oscilloscope.

\section{Stimuli}

The acoustic and electric signals were always presented simultaneously and both had a duration of $1 \mathrm{~s}$. The electric signal was a train of pulses with an interphase gap of $8 \mu$ s and a pulse width of $25 \mu$ s. The stimulation mode was monopolar, using both extracochlear reference electrodes in parallel $(\mathrm{MP} 1+2)$. Electrode numbers will be reported from apex to base, the most apical electrode (A) being electrode 1 . This electrode is expected to correspond to the lowest perceived pitch and the most basal electrode (B; electrode 22) corresponds to the highest perceived pitch. For each subject, measurements were performed on three target electrodes, one at the first quarter of the array, one in the middle, and one at the third quarter of the array. Mostly target electrodes 6 (apical), 11 (middle (M)), and 16 (basal) were used. In preliminary tests, electrode 1 or 2 was also included, but, as the subjects did not show ITD sensitivity with these electrodes, they were not included in the final test protocol.

In the main body of results, an electric pulse train of 100 pps, combined with an acoustic filtered click train was used, unless reported otherwise. The acoustic filtered click train was generated using Matlab by adding individual harmonics whose frequencies were multiples of $100 \mathrm{~Hz}$. The harmonics were added in phase. A discrete set of cutoff frequencies was selected 
for this study, such that the bandwidth of the acoustic signal was one octave. The harmonics used for the acoustic signals were respectively $2-4,4-8,8-16,16-$ 32 , and 32-64, resulting in cutoff frequencies of 200 $-400,400-800,800-1,600,1,600-3,200$, and 3,200 $-6,400 \mathrm{~Hz}$. In preliminary experiments, high-rate (6,300 pps) transposed stimuli were used as well as click trains of 100 and 150 pps.

An example electric and acoustic signal is shown in Figure 1. In this paper, the separate electric or acoustic signals are called "signal" and the combination of an electric and acoustic signal is called "stimulus."

\section{Procedures}

The procedures consist of three main parts: first, $\mathrm{T}$ and $\mathrm{C}$ levels are fitted; then, the loudness between the ears is balanced and finally the JND in ITD is determined using a lateralization paradigm. An overview of the procedures is shown in Figure 2 by means of a fictive example. As ITDs and ILDs both influence the lateralization of a stimulus, great care has to be taken that ITD and ILD are not confused in the procedural design and analysis of the results.

Fitting of $\mathrm{T}$ and $\mathrm{C}$ levels. During the first test session, the target electrodes (A, M, and B) were fitted at 100 pps. First, the hearing threshold $(\mathrm{T})$ was determined and then the comfortable level $(\mathrm{C})$, the loudest level that was not uncomfortable for the subject. In each test session, the fitting of at least one of the electrodes was verified. In all subsequent tests, the comfortable level was never exceeded.
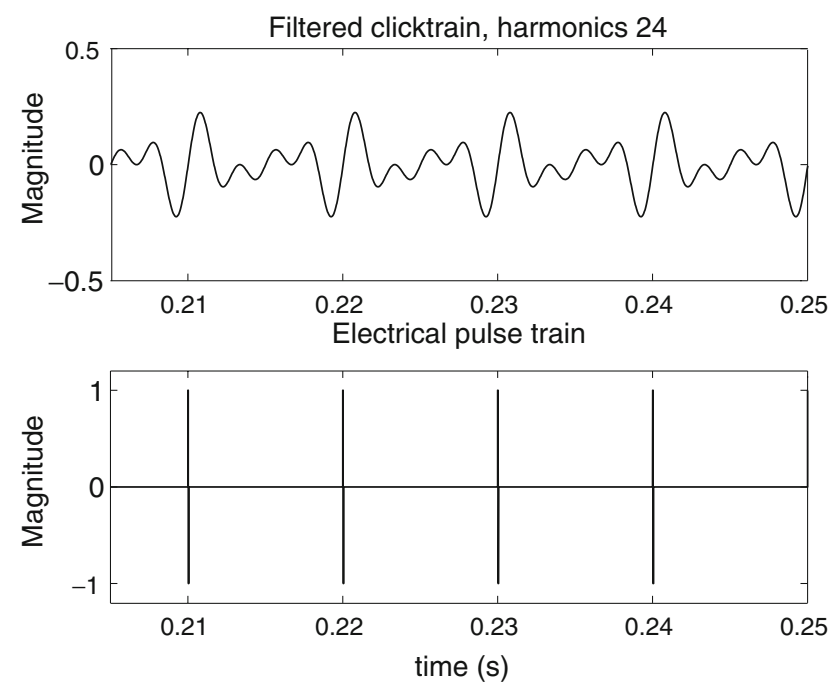

FIG. 1. Part of an example stimulus. The top panel shows a filtered click train with harmonics $2-4$ and $F_{0}=100 \mathrm{~Hz}$ and the bottom panel an electric pulse train of 100 pps.
For every acoustic signal, the hearing threshold and comfortable level was determined at the start of the test session where it was to be used.

Loudness balancing of stimuli. JNDs in ITD in normalhearing subjects are smallest when the ILD is zero. Balancing of ILD was however complicated by the fact that lateralization is influenced by both ILD and ITD, such that the ITD cannot be set to zero because the exact delay to be introduced into the electric path is unknown.

To avoid differences between monaural and binaural stimulation influencing the results, the signals were presented simultaneously at both ears during the loudness-balancing procedures. Loudness balancing was performed in two steps. In the first step, a loudness-balancing experiment was performed with a modified stimulus from which all possible ITD information was removed. In the second step, the balance from step 1 was refined by assessing the extent of lateralization. In subsequent experiments, only the result from step 2 will be used.

The modified stimulus of step 1 was as similar as possible to the target stimulus but with all possible ITD cues removed. The human auditory system can perceive ITDs in the onset part of a stimulus and in the ongoing part (Moore 2003; Laback et al. 2007). The onset and offset cue was removed by using a cosinusoidal ramp of $200 \mathrm{~ms}$, yielding a stationary part of $600 \mathrm{~ms}$. The ongoing cue was removed by jittering the time between the individual pulses, both in the electric and the acoustic signal. The degree of jitter introduced was a parameter that was adjusted to obtain a similar roughness percept at the two ears. ${ }^{1}$

In the subsequent loudness-balancing procedure, the acoustic signal was first set to a comfortable level. Then, in a constant stimuli procedure, the intensity of the electric signal was varied in steps of $10 \%$ of the subject's electric dynamic range. The subject was queried for each stimulus whether the sound in the left or right ear sounded louder. A psychometric function was then fitted to the results (Wichmann and Hill 2001), and the $50 \%$ correct point, yielding the electric intensity corresponding in loudness to the acoustic intensity, was determined.

In step 2, the electric intensity from step 1 was refined by assessing whether the sound could be lateralized

\footnotetext{
${ }^{1}$ As one subject perceived the jittered electric and acoustic signals as dissimilar, a jitter-balancing procedure was first performed to determine the amount of jitter in both signals such that the signals were perceived as similar. The subjects reported that adding jitter to the signals corresponded to a percept of roughness. Therefore, a constant stimuli procedure was used in which the acoustic signal was followed by the electric signal and the subject's task was to indicate which signal sounded the most "rough," the first or second. The result was a percentage of acoustic jitter corresponding in roughness to a percentage of electric jitter.
} 


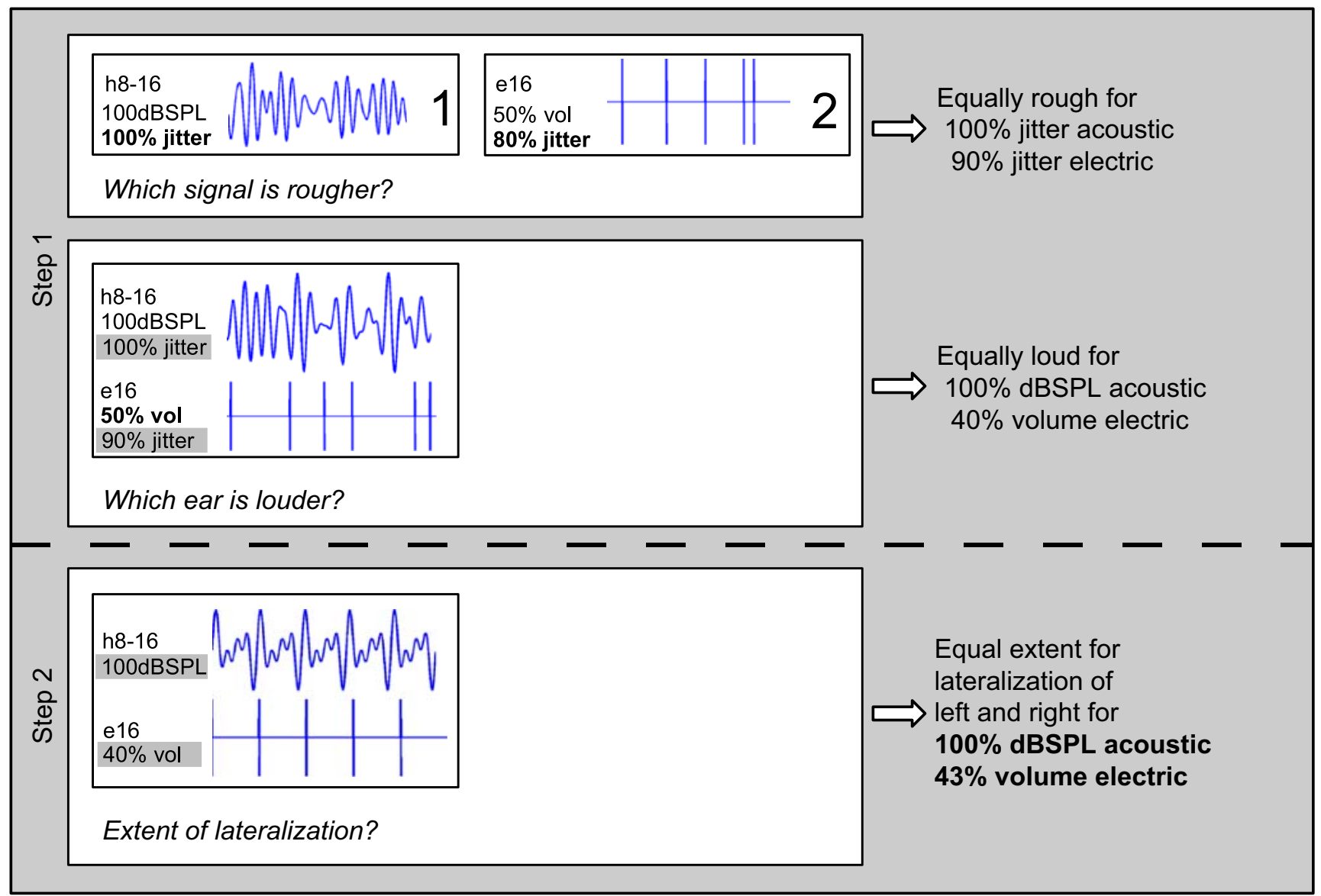

h8-16: harmonics 8 up to $16 ; \quad$ e16: electrode $16 ; \quad 50 \%$ vol: volume of $50 \%$ of the electric dynamic range

FIG. 2. Graphical overview of the used loudness-balancing procedures. The white boxes illustrate the stimuli presented to the subject and the text on the right shows example results from each procedure. Each procedure uses parameters determined in the previous procedure, which are shown with a gray background. The numbers are fictive but of a realistic magnitude. The plotted electric and acoustic signals only serve illustrative purposes and do only show part of example signals.

equally far to the left and right-hand side by varying only the ITD. If necessary, a slight change was made to the intensity of the electric signal. The change was always less than $10 \%$ of the electric dynamic range. In what follows, only the final result from step 2 will be used. Note that, at first, before step 2 could be performed, some familiarization with ITD perception was necessary, ranging from $1 \mathrm{~h}$ up to multiple sessions.

The result of steps 1 and 2 is a stimulus that is balanced in loudness, the equivalent of a stimulus with an ILD of $0 \mathrm{~dB}$ in a normal-hearing subject. Note that these procedures have to be performed again for each change to either the electric signal or acoustic signal.

Measurement of psychometric functions for ITD. Before determination of the JND in ITD, the subjects were slowly trained by at first presenting them stimuli with large ITD cues (up to $3 \mathrm{~ms}$ off center) manually and then using a two-alternative forced-choice procedure to assess ITD discrimination. Feedback was never given.

Then, the psychometric function for ITD was determined using a constant stimuli procedure. A number of ITDs was selected over a certain range and a stimulus containing each ITD was presented three times. The subject had to respond whether the sound was lateralized to the left or right side. The ITDs to be presented in one condition were determined manually based on previous subject performance. Some very large ITDs (up to $1.5 \mathrm{~ms}$ off center) were always included to motivate the subject. In the proximity of the crossover point, the intervals were either 500, 250 , or $100 \mu \mathrm{s}$, based on the subject's performance.

Psychometric functions were then fitted to the results using the psignifit toolbox version 2.5.6 for Matlab (see http://bootstrap-software.org/psignifit/) which implements the maximum-likelihood method described by Wichmann and Hill (2001). The $68 \%$ confidence intervals around the fitted values were 
obtained by the BCA bootstrap method implemented by psignifit, based on 1,999 simulations. Results of a psychometric function were only regarded as valid if a confidence interval could be calculated by the bootstrap method and if there was no perfect separation, i.e., if there were points on the slope of the psychometric function different from 0 and 1 . If the same experiment was performed multiple times during one test session, the results of those experiments were merged into a single psychometric function. An example psychometric function is shown in Figure 3.

From each psychometric function, the JND in ITD was determined as half the difference between the $75 \%$ point and the $25 \%$ point of the psychometric function. A $68 \%$ confidence interval for the JND was determined by the combination of the confidence intervals around these points found by the bootstrap method. If multiple psychometric functions were determined for the same condition (e.g., during different test sessions), the median JND was included in the results.

The ITD at the $50 \%$ point of the psychometric function indicates the point where both signals are received synchronously at the auditory nerve. The acoustic signal travels through the middle ear and part of the inner ear before nerve fibers are stimulated. Therefore, the electric signal has to be delayed. The travel time of the acoustic signal depends on its frequency content: lower frequencies have a larger traveling wave delay in the cochlea. This point corresponds to what

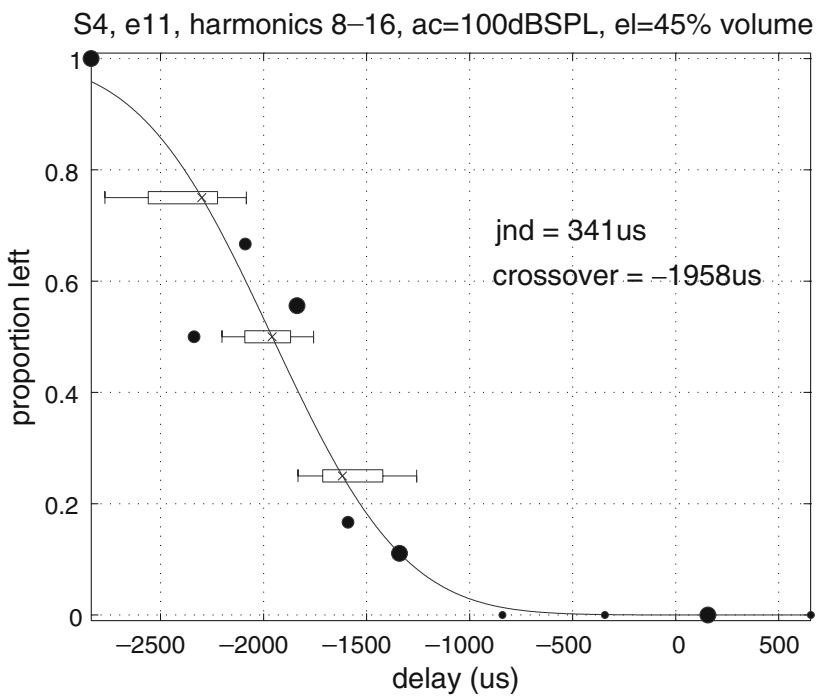

FIG. 3. Example psychometric function for $\mathrm{S} 4$ used to determine the JND in ITD and delay required for psychoacoustically synchronous stimulation using electrode 11 and harmonics $8-16$ for the acoustic signal. The level of the acoustic signal was 100-dB SPL and the level of the electric signal was $45 \%$ of the dynamic range. The found crossover point $(-1,958 \mu \mathrm{s})$ corresponds to $D_{\mathrm{e}}$. For the measurement of this psychometric function, a total of 63 trials were used.

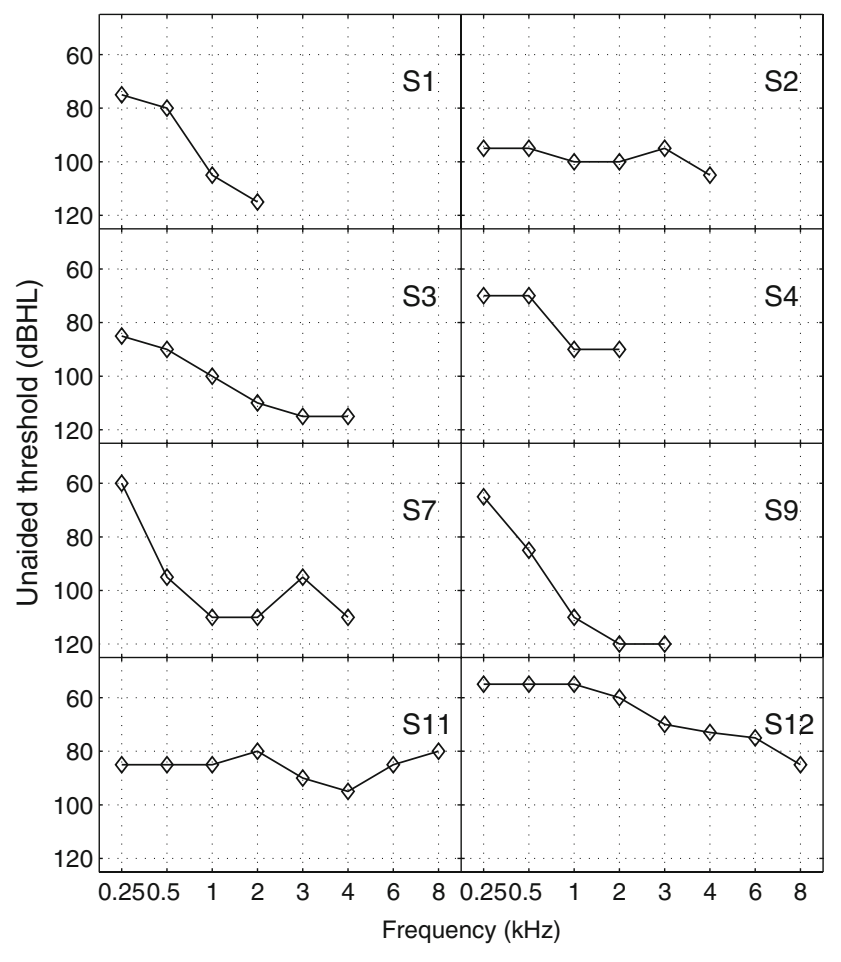

FIG. 4. Unaided pure-tone audiograms per subject as measured during routine audiometry. Note that the vertical axis starts at $60 \mathrm{dBHL}$. If no marker is shown, no threshold could be measured using the clinical audiometry equipment.

would in NH subjects be ITD $=0 \mu$ s. In what follows, this value will be called $D_{\mathrm{e}}$ and is expressed in microsecond delay of the electric signal versus the acoustic signal. Domnitz (1973) and Shepard and Colburn (1976) show that in normal-hearing subjects JNDs in ITD are smallest when the ILD is zero. Whenever it was unclear what the correct loudness balance was in the current study, the JND in ITD was measured for different balances and $D_{\mathrm{e}}$ for which the JND was the smallest was reported.

\section{Subjects}

All subjects were recruited among the clinical population of the University Hospital Maastricht (AZM) and the University Hospital Leuven (UZLeuven). They were volunteers and signed an informed consent form. This study was approved by the local medical ethical committees. All subjects wore an HA contralaterally to their CI on a daily basis and used a CI of the Nucleus24 type (Cochlear Ltd). S1 and S12 had an electrode array of the Contour Advance type; the other subjects had an array of the Contour type. The clinical processors were of the ESPrit3G type for all but two subjects, who used a Freedom processor instead. All unaided pure-tone audiograms as measured during routine audiometry are shown in Figure 4. 
In a previous study (Francart et al. 2008a), sensitivity to ILDs was measured in ten subjects. Of those subjects, six were selected for the current study, based on their availability and their ability in performing psychophysical tasks. Two other subjects were included who were implanted more recently (S11 and S12). Relevant data for all eight participating subjects are shown in Table 1.

The subjects came to the hospital for 2 up to 12 test sessions of about $2 \mathrm{~h}$ with at least 1-week time and maximally 1-month time between sessions.

Subject S9 had an incomplete electrode array insertion in the cochlea with two electrodes lying outside of the cochlea. All other subjects had normal electrode insertions.

\section{RESULTS}

\section{Loudness balancing and intensity}

The loudness-balancing task with the jittered stimulus was at first somewhat confusing for the subjects. Because the stimulus yielded a diffuse image and could not easily be lateralized, they had to consciously compare loudness differences between the ears. As a result, performance was somewhat lower than on the loudness-balancing tasks in our previous study (Francart et al. 2008a).

When repeating the balancing experiments within the same session, the balancing results were quasiidentical. However, between-session differences were observed, mostly in the order of $5 \%$ or $10 \%$ of the electric dynamic range. Possibly, this was correlated with temporary threshold shifts in the residual hearing.

For all subjects except S2, the acoustic signals could be set to a comfortable level. S2 rated the maximal output level of the transducer as "too soft" and no sensitivity to ITD could be observed with the soft signals. Therefore, the bandwidth of the acoustic signals was halved, for example, using harmonics
16-23 and 23-32 instead of 16-32, yielding a maximal output level that was comfortably loud.

\section{JND in ITD}

None of the subjects could at first perceive differences in ITD, even when using stimuli that proved successful later on. This is not surprising as these ITD cues are probably not available with their own speech processor in combination with their HA. After training, they reported hearing the stimulus at the back of the head, where it shifted to the left or right according to the ITD.

In several cases, at the beginning of a new test session, subjects could not consistently lateralize stimuli which they could lateralize during the previous test session. Therefore, at the beginning of every new test session, the subjects were trained by presenting them stimuli with large ITD cues. It seems that they did not have a frame of reference for ITD cues and had to be "recalibrated" every test session.

In preliminary experiments, perception of ITDs could be achieved using the stimulus containing transposed signals and the stimulus containing an electric pulse train and an acoustic filtered click train. Of the latter stimulus, both 100 and 150 pps were assessed. As subject S4 could not perceive ITDs with the 150-pps stimulus, this condition was not included in any further tests. As the bandwidth of the filtered click train can be varied and 100-pps click trains are used in many ITD-CI studies, only the 100-pps click train was used for the final experiments.

Consistent data of the JND in ITD could be collected for four out of eight subjects. In Figure 5, JNDs are reported per subject for each combination of the acoustic and electric signals that yielded valid JNDs. Each box corresponds to a condition, i.e., a combination of electric and acoustic signal, and the value reported is the median JND in microseconds that was found for that condition over the different test sessions. If the JND in ITD could not be measured

TABLE 1

\begin{tabular}{|c|c|c|c|c|c|c|c|c|}
\hline \multicolumn{9}{|c|}{ Subject information } \\
\hline Subject & Age & Months of use & $\mathrm{Cl}$ side & Etiology & Apex & Middle & Base & Performance \\
\hline S1 & 58 & 42 & $\mathrm{R}$ & Progressive & 6 & 11 & 16 & None \\
\hline S2 & 66 & 49 & $\mathrm{R}$ & Noise exposure & 6 & 10 & 16 & Good \\
\hline S3 & 68 & 58 & $\mathrm{R}$ & Ménière & 6 & 11 & 16 & None \\
\hline S4 & 76 & 45 & $\mathrm{R}$ & Progressive & 6 & 11 & 16 & Good \\
\hline S7 & 40 & 71 & $\mathrm{R}$ & Meningitis & 6 & 11 & 16 & Poor \\
\hline S9 & 32 & 70 & $\mathrm{~L}$ & Autoimmune & 1 & 6 & 11 & Poor \\
\hline S11 & 62 & 25 & $\mathrm{R}$ & Ménière & 6 & 11 & 16 & Good \\
\hline S12 & 65 & 5 & $\mathrm{~L}$ & Genetic (DFNA9) & 6 & 11 & 16 & Good \\
\hline
\end{tabular}

Age is in years at the time of testing. Months of use is the number of months of implant use at the time of testing. $\mathrm{Cl}$ side is left (L) or right (R). The $\mathrm{HA}$ was on the other side. Performance is the category of ITD perception performance. Apex, Middle, and Base are the tested electrodes at apical, medial, and basal positions in the electrode array 

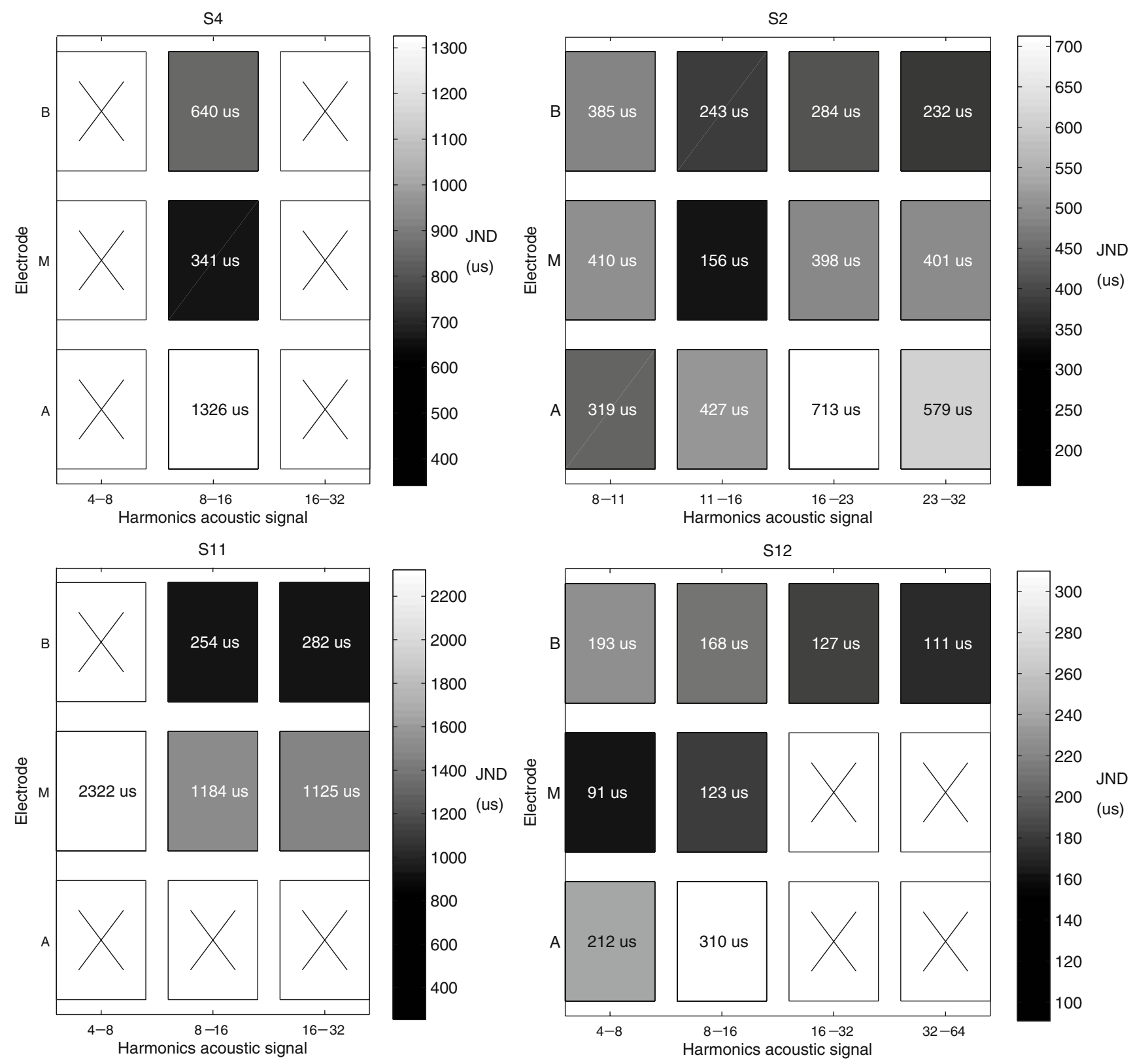

FIG. 5. Median JND in ITD in microsecond per subject per condition. A cross indicates that the condition was tested but that sensitivity to ITD was insufficient to do the lateralization task.

due to insufficient sensitivity to ITD, the condition is marked with a cross.

Figure 6 shows the median JND in ITD per subject and electrode for the acoustic signal that yielded the lowest JND in ITD. Assuming that ITD perception is best for signals matched in place in the cochlea, it therefore shows the JND in ITD for place-matched stimulation per subject and electrode. For comparison, reference values from the bilateral CI literature for pulse trains of 100 pps are given above the label "2x CI". Additionally, Table 2 lists the acoustic signal for which performance was best per electrode. In total, 87 psychometric functions were determined for which performance was better than chance level. Each of them was determined based on between 21 and 117 trials.

It should be noted that while the best-reported median JNDs per subject are in the order of 100$350 \mu \mathrm{s}$, JNDs were measured as low as respectively 57 , 91, 155, and $91 \mu$ s for subjects S2, S4, S11, and S12.

For each subject, we determined whether their ITD perception performance was none, poor, or good. Subjects in category none could not detect any ITD at all. Subjects in category poor seemed to be able to 


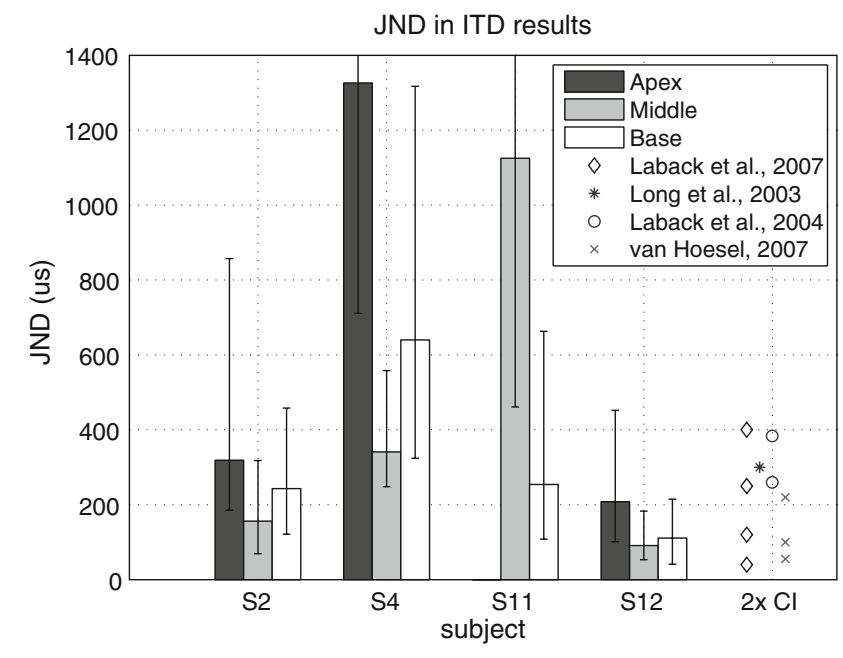

FIG. 6. Best median JND in ITD per subject and per electrode. The values above the label $2 x \mathrm{Cl}$ are reference values from the bilateral $\mathrm{Cl}$ literature for pulse trains of 100 pps. Each symbol is the JND in ITD for one subject. The error bars are $68 \%$ confidence intervals on the fit of the psychometric function, determined by a bootstrap method.

detect large differences in ITD in informal tests but could not consistently lateralize using only ITD cues. Subjects in category good could both detect ITD differences and lateralize using ITDs. In Figure 7, the three categories are plotted versus the thresholds of the residual hearing of each subject. Whether a subject is in category good is related with the average threshold at 1,000 and 2,000 Hz. A Wilcoxon rank-sum test between category good and categories none-poor shows a significant effect $(p=0.03)$.

\section{Delays}

In addition to the JND in ITD, the psychometric function also indicates $D_{\mathrm{e}}$, the point where both signals are received synchronously at the auditory nerve (see "Measurement of psychometric functions for ITD"). Figure 8 shows a histogram of delays

\section{TABLE 2}

Range of harmonics of best-matching acoustic signal for each electrode per subject

\begin{tabular}{llll}
\hline Subject & Apex & \multicolumn{1}{c}{ Middle } & \multicolumn{1}{c}{ Base } \\
\hline S2 & $8-11$ & $11-16$ & $23-32$ \\
S4 & $8-16$ & $8-16$ & $8-16$ \\
S7 & N/A & N/A & $8-16$ \\
S9 & N/A & $8-16$ & N/A \\
S11 & N/A & $8-16 / 16-32$ & $8-16 / 16-32$ \\
S12 & 4-8 & $4-8$ & $32-64$ \\
\hline
\end{tabular}

If there was no considerable difference between several acoustic signals, both are given
ITD detection performance versus audiometric thresholds

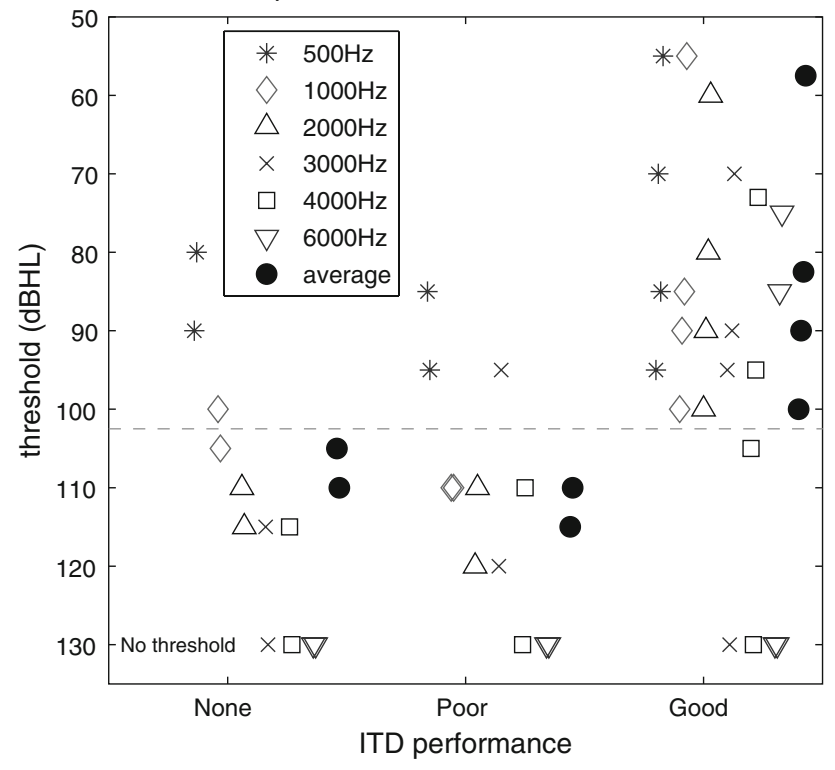

FIG. 7. ITD perception performance versus thresholds of residual hearing. Each different marker denotes a different threshold measurement frequency. The filled circles show the average threshold at frequencies 1,000 and $2,000 \mathrm{~Hz}$.

encountered in all experiments. The median is $1.5 \mathrm{~ms}$. Our data show that $D_{\mathrm{e}}$ depends on the ILD. When ITD perception performance was low, it was entirely disrupted by introduction of a nonzero ILD. When performance was high, $D_{\mathrm{e}}$ depended on the ILD, such that the ear with the louder signal had to be delayed versus the other ear for the stimulus to be

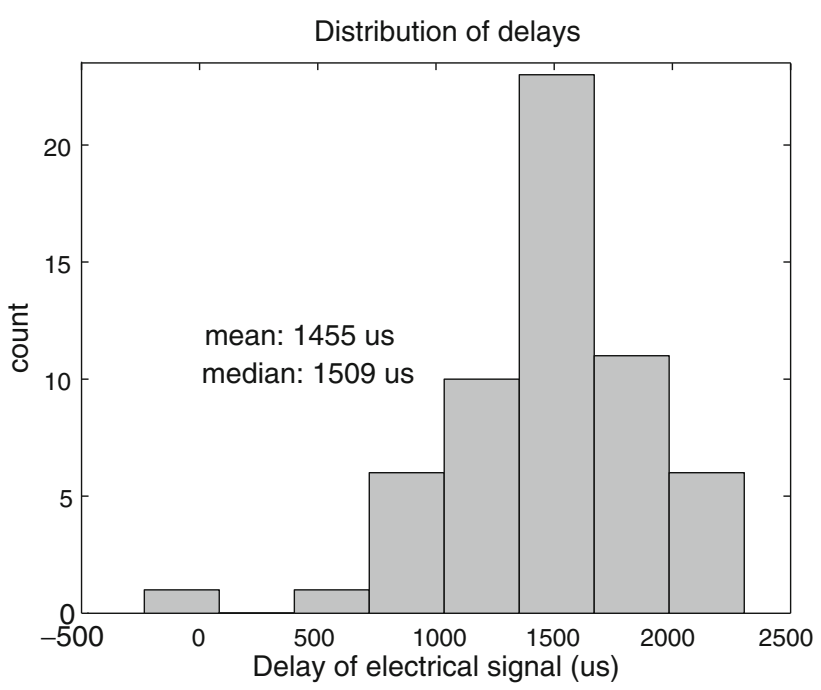

FIG. 8. Histogram of $D_{\mathrm{e}}$ values. Each value contributing to an increment of one on the vertical axis corresponds to a value found by fitting a psychometric function to the response to between 21 and 117 trials. If measurements with the same stimulus were available at different ILDs, only the $D_{\mathrm{e}}$ was selected for which the corresponding JND in ITD was smallest. 
centered perceptually. If for example one holds the amplitude of the acoustic signal constant and increases the amplitude of the electric signal, $D_{\mathrm{e}}$ also increases. This phenomenon is also observed in normal-hearing subjects and is often called timeintensity trading (Moore 2003).

Because the traveling wave delay in the cochlea increases with decreasing frequency, one would expect $D_{\mathrm{e}}$ to vary with the frequency content of the acoustic signal. However, when changing the acoustic signal, the balancing procedure had to be repeated, yielding, possibly, a slightly different balance, which influences $D_{\mathrm{e}}$ and thus confounds the comparison between different stimuli. A clear tendency of change in $D_{\mathrm{e}}$ with changing frequency content is not observed in our data due to (1) balancing differences, (2) the largest possible change in frequency content being severely limited by the amount of residual hearing, and (3) the subjects not being sensitive to ITD using the most apical electrodes of the CI.

\section{Matching the place of excitation}

Assuming that ITD perception is best for signals bilaterally matched in place of excitation in the cochlea (Nuetzel and Hafter 1981), the best match in place can be determined by considering the minimum JND in ITD for several acoustic frequencies. For subjects S4 and S11, there are not enough data available, but, considering Figure 5 for subjects S2 and S12, there is a tendency of increasing best acoustic frequency per increasing electrode number. For S2, electrode 6 performance is best for harmonics $8-11$; electrode 10 corresponds to harmonics 11-16 and electrode 16 to harmonics 23-32. For S12, electrode 6 corresponds to harmonics 4-8, electrode 11 to harmonics 4-8 (but with a lower JND than for electrode 6), and electrode 16 corresponds to harmonics 32-64.

\section{DISCUSSION}

\section{Influence of ILD}

The sensitivity to ILD of users of a bimodal hearing system approaches that of normal-hearing listeners (Francart et al. 2008a), but their dynamic range is much smaller. Therefore, small differences in ILD can have large perceptual consequences. ITD perception in NH subjects is optimal when the ILD is zero (Domnitz 1973; Shepard and Colburn 1976). Therefore, in the current study, loudness differences between the ears were eliminated as much as possible. Whenever determination of the JND in ITD was attempted with levels deviating from those according to the results of the loudness-balancing procedures, the measurement did not succeed or yielded a large
JND. An adjustment of 5\% of the electric dynamic range, corresponding to one or two clinical current units, often made the difference between being able to lateralize using ITD or not.

\section{JND in ITD}

The reported JNDs in ITD are poor in comparison to NH listeners (Bernstein and Trahiotis 2002) and of the same order of magnitude as the values found for bilateral CI users (Senn et al. 2005; Long et al. 2003; Majdak et al. 2006; Laback et al. 2004, 2007; van Hoesel 2004, 2007; Lawson et al. 1998; van Hoesel and Tyler 2003). While JNDs in ITD of around 100-350 $\mu \mathrm{s}$ are poor compared to the best fine-structure JND in ITD of $10 \mu$ s found in NH subjects (Yost 1974), they are comparable to envelope JND in ITD found in NH subjects for the same rate in the modulator (Bernstein and Trahiotis 2002). Moreover, it should be noted that the residual hearing of our subjects was rather limited relative to that of many subjects who are nowadays receiving a cochlear implant and possibly better performance may be achieved with better residual hearing.

Our data do not only demonstrate ITD perception capability but also lateralization capability. The sound image was clearly steered to the left or right side when introducing ITDs, after carefully balancing the signals in loudness. This means that, if clear ITD cues could be transmitted by the CI and HA, they could be used for localization of sound sources and provide advantages such as binaural unmasking, which is very important for speech perception in noise. For ITDs to be transmitted by a real CI and HA, both devices must be carefully balanced in loudness and the cutoff frequencies of the band pass filter corresponding to each electrode must be approximately matched to the corresponding acoustic signal in the other ear.

Most probably, only onset and envelope ITD cues were used by the bimodal subjects in this study, considering (1) that JNDs for NH listeners with amplitude-modulated signals are comparable to the values found here (Bernstein and Trahiotis 2002), (2) that CI users are mainly reported to use envelope cues for ITD perception and could therefore be assumed to perceive ITDs using the neural mechanisms that $\mathrm{NH}$ listeners use for envelope ITD perception, and (3) the type of signals used in this study. In preliminary experiments, no sensitivity to ITD was found at a more apical electrode or lower acoustic frequencies than reported and in most cases our data show best ITD perception performance for acoustic signals with cutoff frequencies of 800-1,600 and 1,600-3,200 Hz. As ITDs in the fine structure of an acoustic signal can only be detected up to about $1.3 \mathrm{kHz}$ (Zwislocki and Feldman 1956), this is indicative for 
perception of ITD in the envelope instead of in the fine structure of the signals. This is probably related to our finding that ITD perception performance of bimodal subjects is related with the average thresholds of their residual hearing at 1,000 and $2,000 \mathrm{~Hz}$ and not with the thresholds at lower frequencies. The reason for the subjects' apparent inability to use fine-structure ITD cues is currently unclear.

By means of the binaural interaction component in animal models, Noh et al. (2007) have shown that the binaural auditory system can process combinations of electric and acoustic stimulation across ears. This is confirmed by our finding that users of a bimodal system can detect ITDs.

\section{Delays}

The median delay to be introduced into the electric pathway for psychoacoustically synchronous stimulation was $1.5 \mathrm{~ms}$. This is the first report of transmission delay between electric and acoustic stimulation. The value of this delay is comparable to the difference between the delays obtained from the acoustic auditory brain stem response (ABR) and electric auditory brain stem response (EABR) literature. While the paradigms and experimental setups differ between studies, the reported latencies are similar.

In Table 3, wave $\mathrm{V}$ latencies for different studies are summarized. Don and Eggermont (1978) show that all frequency regions contribute to the ABR but that the response is dominated by contributions from the two to three octaves towards the basal end of the cochlea. Therefore, the values in the "base" column of Table 3 are compared to the acoustic ABR values. On average, the difference is $1.5 \mathrm{~ms}$, which, given the procedural- and presentation-level differences between studies, corresponds well to the 1.5 -ms latency difference found in the current study.

TABLE 3

Wave $\mathrm{V}$ latencies (in millisecond) from different studies on $\mathrm{ABR}$ and $\mathrm{EABR}$

\begin{tabular}{llll}
\hline \multirow{2}{*}{ Publication } & \multicolumn{3}{c}{ Stimulus } \\
\cline { 2 - 4 } & Apex & Mid & Base \\
\hline Electric click & & & \\
Shallop et al. (1990) & 3.82 & 3.94 & 4.20 \\
Abbas and Brown (1991) & 3.87 & 3.90 & 4.12 \\
\hline
\end{tabular}

Acoustic click

Nikiforidis et al. (1993) $\quad 5.64$

UZLeuven $\quad$ man 5.78 , woman 5.57

All measured at a comfortably loud level. The last row shows reference values used for the clinical ABR setup in our hospital (UZLeuven)
Relation with localization performance and binaural unmasking

In the current study, all parameters were optimized as to achieve optimal ITD sensitivity. It is therefore unlikely that users of current clinical CIs and HAs would be able to benefit directly from ITD cues, also given the problems with current clinical devices enumerated in the introduction.

In preliminary tests, our subjects did show similar ITD sensitivity using transposed stimuli. The electric signal was a high-rate pulse train $(6,300 \mathrm{pps})$ transposed with a modulation frequency of $42 \mathrm{~Hz}$. The acoustic signal was a sinusoid matched in pitch with the unmodulated electric pulse train, also transposed with $42 \mathrm{~Hz}$. This is comparable to the situation with clinical devices where intermediate to high pulse rates are used and signals with slow modulations (such as speech) are presented. Therefore, for the four subjects who showed ITD sensitivity, it might be possible to use interaural timing cues in real-world signals if the CI and HA signal processing and fitting is modified to achieve (1) correct binaural loudness growth (Francart et al. 2008a), (2) correct synchronization, and (3) correct matching of the places of excitation in the cochleas.

The perception of binaural timing cues can give rise to improved sound localization performance and more importantly binaural unmasking of speech in background noise.

\section{Conclusions}

If the average threshold of the residual hearing at 1,000 and $2,000 \mathrm{~Hz}$ is better than about $100 \mathrm{dBHL}$, lateralization is possible with ITD cues for subjects using a CI in one ear and an HA in the other. The best median JNDs in ITD were 156, 341, 254, and $91 \mu$ s in the four of the eight subjects who could discriminate ITDs. This is comparable to the values found in the literature on bilateral CIs. ITDs could in most cases only be detected at acoustic frequencies above about $1 \mathrm{kHz}$. This indicates that mainly envelope cues were used. For the acoustic and electric signals to be perceived synchronously, the electric signal should be delayed by $1.5 \mathrm{~ms}$.

\section{ACKNOWLEDGEMENTS}

This research was carried out in the frame of the IWT (Institute for the Promotion of Innovation by Science and Technology in Flanders) project 050445 and was partly sponsored by Cochlear Ltd. We thank our test subjects for their patient and enthusiastic participation in the experiments. We also thank the staff of the audiology department of the hospital of Maastricht and the hospital of Leuven for their 
kind cooperation and flexibility. We gratefully acknowledge the help of Bas van Dijk and Clemens Zweekhorst for providing the necessary hardware and their help with our test setup.

\section{REFERENCES}

Aвbas P, Brown C. Electrically evoked auditory brainstem response: growth of response with current level. Hear. Res. 51(1):123-137, 1991.

Bernstein L, Trahiotis C. Lateralization of low-frequency, complex waveforms: the use of envelope-based temporal disparities. J. Acoust. Soc. Am. 77(5):1868-1880, 1985.

Bernstein L, Trahiotis C. Enhancing sensitivity to interaural delays at high frequencies by using "transposed stimuli". J. Acoust. Soc. Am. 112(3 Pt 1):1026-1036, 2002.

Blanks D, Roberts J, Buss E, Hall J, Fitzpatrick D. Neural and behavioral sensitivity to interaural time differences using amplitude modulated tones with mismatched carrier frequencies. J. Assoc. Res. Otolaryngol. 8(3):393-408, 2007.

Ching T, van Wankooy E, Dillon H. Binaural-bimodal fitting or bilateral implantation for managing severe to profound deafness: a review. Trends Amplif. 11(3):161-192, 2007.

DomNiTz R. The interaural time JND as a simultaneous function of interaural time and interaural amplitude. J. Acoust. Soc. Am. 53 (6):1549-1552, 1973.

Don M, Eggermont J. Analysis of the click-evoked brainstem potentials in man using high-pass noise masking. J. Acoust. Soc. Am. 63(4):1084-1092, 1978.

Francart T, Brokx J, Wouters J. Sensitivity to interaural level difference and loudness growth with bilateral bimodal stimulation. Audiol. Neurootol. 13(5):309-319, 2008a.

Francart T, van Wieringen A, Wouters J. APEX 3: a multi-purpose test platform for auditory psychophysical experiments. J. Neurosci. Methods 172(2):283-293, 2008b.

HAFTER E, CARRIER S. Binaural interaction in low-frequency stimuli: the inability to trade time and intensity completely. J. Acoust. Soc. Am. 51(6):1852-1862, 1972.

Henning G. Detectability of interaural delay in high-frequency complex waveforms. J. Acoust. Soc. Am. 55(1):84-90, 1974.

Laback B, Pok S, Baumgartner W, Deutsch W, Schmid K. Sensitivity to interaural level and envelope time differences of two bilateral cochlear implant listeners using clinical sound processors. Ear Hear 25(5):488-500, 2004.

LABACK B, MAJDAK P, BAUMGARTNER W. Lateralization discrimination of interaural time delays in four-pulse sequences in electric and acoustic hearing. J. Acoust. Soc. Am. 121(4):2182-2191, 2007.
Lawson D, Wilson B, Zerbi M, van den Honert C, Finley C, Farmer J, Jr, McElveen J, JR, Roush P. Bilateral cochlear implants controlled by a single speech processor. Am. J. Otol. 19 (6): 758-761, 1998.

Long C, EdDington D, Colburn H, Rabinowitz W. Binaural sensitivity as a function of interaural electrode position with a bilateral cochlear implant user. J. Acoust. Soc. Am. 114(3):1565-1574, 2003.

MajDaK P, Laback B, Baumgartner W. Effects of interaural time differences in fine structure and envelope on lateral discrimination in electric hearing. J. Acoust. Soc. Am. 120(4):2190-2201, 2006.

Moore B. An Introduction to the Psychology of Hearing, 5th edition. Amsterdam, Elsevier Science, 2003.

Nikiforidis G, Koutsojannis C, Varakis J, Goumas P. Reduced variance in the latency and amplitude of the fifth wave of auditory brain stem response after normalization for head size. Ear Hear 14 (6):423-428, 1993.

Noh H, Abbas P, Abbas C, Nourski K, Robinson B, Jeng F. Binaural interactions of electrically and acoustically evoked responses recorded from the inferior colliculus of guinea pigs. Int. J. Audiol. 46(6):309-320, 2007.

Nuetzel J, Hafter E. Discrimination of interaural delays in complex waveforms: spectral effects. J. Acoust. Soc. Am. 69(4):1112-1118, 1981.

Senn P, Kompis M, Vischer M, Haeusler R. Minimum audible angle, just noticeable interaural differences and speech intelligibility with bilateral cochlear implants using clinical speech processors. Audiol. Neurootol. 10(6):342-352, 2005.

Shallop J, Beiter A, Goin D, Mischke R. Electrically evoked auditory brain stem responses (EABR) and middle latency responses (EMLR) obtained from patients with the nucleus multichannel cochlear implant. Ear Hear 11(1):5-15, 1990.

SHEPARD N, Colburn S. Interaural time discrimination of clicks: dependence on interaural time and intensity differences. J. Acoust. Soc. Am. 59(S1):S23, 1976.

VAN HOESEL R. Exploring the benefits of bilateral cochlear implants. Audiol. Neurootol. 9(4):234-246, 2004.

vaN HoEsel R. Sensitivity to binaural timing in bilateral cochlear implant users. J. Acoust. Soc. Am. 121(4):2192-2206, 2007.

van Hoesel R, Tyler R. Speech perception, localization, and lateralization with bilateral cochlear implants. J. Acoust. Soc. Am. 113(3):1617-1630, 2003.

Wichmann F, Hill N. The psychometric function: II. Bootstrap-based confidence intervals and sampling. Percept. Psychophys. 63 (8):1314-1329, 2001.

Yost W. Discriminations of interaural phase differences. J. Acoust. Soc. Am. 55(6):1299-1303, 1974.

Yost W, Wightman F, Green DM. Lateralization of filtered clicks. J. Acoust. Soc. Am. 50(6B):1526-1531, 1971.

Zwislocki J, Feldman R. Just noticeable differences in dichotic phase. J. Acoust. Soc. Am. 28(5):860-864, 1956. 